\title{
Change advice on antibiotics with caution
}

\author{
The recommendation that antibiotic courses are always completed should be dropped according to a recent \\ analysis. While a welcome addition to discussion on the role of stewardship in tackling resistance, caution \\ should be applied before advice on prescription practices and communication with patients is altered.
}

The diagnosis of an infection, whether in ourselves or a loved one, can be a stressful event, especially in light of the on-going spread of multiple drug resistance. Expecting patients to be able to assimilate all of the information necessary to make an educated choice under such high-pressured circumstances is unrealistic. As such, it is essential that we are able to rely on our physicians to recommend appropriate courses of action to treat the infection as rapidly as possible, and to communicate that advice to patients at a level appropriate to the circumstances. Reducing the immediate threat from the infecting microorganism must remain the primary driver behind any therapeutic course; however, it is not the only consideration that must be taken into account when prescribing antimicrobial drugs. The more that antibiotics are used in a population, or an individual, the greater the risk that resistance can be selected for, whether in the primary pathogen itself, or in the rest of the microbial communities associated with patients. Limiting the use of antibiotics to only those cases in which they are necessary and likely to be effective is therefore an important part of antimicrobial stewardship. As argued by Llewelyn and colleagues in a recent analysis ${ }^{1}$ published in the British Medical Journal, the duration for which an antibiotic course is adhered to is an underappreciated yet similarly important factor that also needs to be accounted for when improving stewardship.

The prevailing recommendations from national and international healthcare and education bodies hold that an antibiotic course should always be completed and that failure to do so will increase the risk of selecting for resistance. Yet as Llewelyn et al. compellingly argue, the 'complete the course' concept is based in part on a historic misunderstanding of relapse following the halting of penicillin treatment of a patient with staphylococcal sepsis in 1941, and that for most indications the actual evidence behind this assertion is flimsy or nonexistent. In the identified cases where it was possible to assess the impact of the length of the treatment course, reducing the duration of antibiotic treatment by several days from the standard courses, which often last a week or two, only led to increased clinical failure in a few cases (such as in treating otitis media). Importantly, no study demonstrated increased resistance as a result. In the absence of strong supporting evidence, the authors make the point that 'completing the course' should therefore no longer be advocated when training physicians or communicating with the public. They also urge that the lack of evidence for the current duration of courses should be publicly acknowledged and that further research is needed to better establish the most appropriate alternative messages to be used publicly - they suggest considering 'stop when you feel better'.

While bringing these ideas into discussions about best practice for antimicrobial stewardship is an important and welcome step, great caution should be used to avoid unintended and potentially harmful consequences. Already in the coverage received by this provocative analysis, mainstream news outlets have led with irresponsible headlines advising patients not to finish their course of antibiotics (for example, "Don't finish the course of antibiotics' - experts turn medical advice on its head", The Telegraph) ${ }^{2}$. Even if somewhat balanced by counter point within the text of an article, such propagandist headlines sow unnecessary doubt among potentially vulnerable patients and risk undermining trust in doctors and healthcare experts. They also provide succour to those who prey on concerns about mainstream medicine to push their preferred quackery, which is potentially highly dangerous to infected individuals already at risk. Furthermore, the suggestion that patients take control of their own treatment course and stop taking antibiotics once they feel better is the proverbial can of worms. Feeling better is clearly subjective; what it means will vary greatly from patient to patient and will not always coincide with clearance of the problem pathogen, which could re-establish infection following a premature cessation of treatment. In addition, when a patient starts to feel better, but has perhaps not recovered completely, if they have been empowered to take control of their own treatment they may arbitrarily choose to reduce the dose of antibiotic being taken rather than stop altogether, a potentially far worse outcome if reducing selection for resistance is the goal. Furthermore, what are the consequences if patients apply the message of greater individual responsibility for controlling their medications to those for chronic metabolic or cardiovascular disease, or for mental health issues?

Rather than relying only on feeling better as a guide for stopping medication, more prudent would be to change current approaches to monitoring markers of infection during a treatment course, to help identify a clinically appropriate point for cessation. It is not uncommon for markers in blood, urine, sputum and other bodily fluids to be assessed, and cultures grown, at the initial diagnosis stage and then not again until the success of the antibiotic course is assessed a week or more later. Introducing new rapid diagnostic tests and increasing the frequency of testing to give a daily readout of infection status would be feasible in a hospital setting, although it will come with a significant additional burden in terms of cost and healthcare personnel requirements. Outside of the hospital, a higher frequency of monitoring is much harder to achieve. As such, if we are to look at reducing the length of antibiotic courses, it will be critical to carry out clinical trials to assess the minimal necessary duration needed for all antibiotics in our arsenal, taking into account factors that vary between patient including age, weight and gender, as well as the type of infecting organism and specific nature of a given indication.

Determining the reasonable boundaries for treatment duration and avoiding making decisions based on assumptions (whether for longer or shorter courses), will help to reduce the overall volume of antibiotics being used, which in turn may help to prolong the emergence of resistance against a given drug. However, patient well-being should not be sacrificed in achieving this aim, nor should their confidence in the medical advice that they receive.

\footnotetext{
References

1. Llewelyn, M. J. et al. BMJ 358, j3418 (2017).

2. Knapton, S. 'Don't finish the course of antibiotics' - experts turn medical advice on its head. The Telegraph (27 July 2017); http://www.telegraph.co.uk/science/2017/07/26/gps-must-stoptelling-patients-finish-course-antibiotics-say/
} 